\title{
Selection and optimization of nutritional risk screening tools for esophageal cancer patients in China
}

\author{
Wen Dong ${ }^{1 *}$, Xiguang Liu' ${ }^{1 *}$, Shunfang $\mathrm{Zhu}^{2 *}$, Di Lu', Kaican Cai ${ }^{1}$, Ruijun Cai ${ }^{1}$, Qing $\mathrm{Li}^{3}$, Jingjing Zeng ${ }^{1}$ and Mei $\mathrm{Li}^{1 \mathrm{~S}}$ \\ 'Department of Thoracic Surgery, Nanfang Hospital, Southern Medical University, Guangzhou, 510515, China \\ ${ }^{2}$ Department of Respiratory Medicine, Nanfang Hospital, Southern Medical University, Guangzhou, 510515, China \\ ${ }^{3}$ Department of Nutrition, Nanfang Hospital, Southern Medical University, Guangzhou, 510515, China
}

BACKGROUND/OBJECTIVES: Malnutrition has multiple impacts on surgical success, postoperative complications, duration of hospital stay, and costs, particularly for cancer patients. There are various nutrition risk screening tools available for clinical use. Herein, we aim to determine the most appropriate nutritional risk screening system for esophageal cancer (EC) patients in China. SUBJECTS/METHODS: In total, 138 EC patients were enrolled in this study and evaluated by experienced nurses using three different nutritional screening tools, the Nutrition Risk Screening 2002 tool (NRS2002), the Patient-generated Subjective Globe Assessment (PG-SGA), and the Nutrition Risk Index (NRI).We compared sensitivity, specificity, positive and negative likelihood ratios, and Youden index generated by each of the three screening tools. Finally, cut-off points for all three tools were re-defined to optimize and validate the best nutritional risk screening tool for assessing EC patients.

RESULTS: Our data suggested that all three screening tools were $100 \%$ sensitive for EC patients, while the specificities were $44.4 \%, 2.96 \%$, and $59.26 \%$ for NRS 2002, PG-SGA, and NRI, respectively. NRI had a higher positive likelihood ratio as well as a higher area under the receiver operating characteristic curve compared to those of NRS 2002 and PG-SGA; although, all three tools had null negative likelihood ratios. After adjusting the cut-off points, the specificity and accuracy for all tools were significantly improved, however, the NRI remained the most appropriate nutritional risk screening system for EC patients. CONCLUSIONS: The NRI is the most suitable (highest sensitivity and accuracy) nutritional risk screening tool for EC patients. The performance of the NRI can be significantly improved if the cut-off point is modified according to the results obtained using MedCalc software.

Nutrition Research and Practice 2020;14(1):20-24; https://doi.org/10.4162/nrp.2020.14.1.20; pISSN 1976-1457 elSSN 2005-6168

Keywords: Nutritional risk screening, esophageal cancer, NRI, Youden index

\section{INTRODUCTION}

Esophageal cancer (EC) is one of the most prevalent cancers worldwide with approximately 500,000 new cases and 406,000 death cases reported every year [1]. Currently, surgery, chemotherapy, radiation, and limited targeted therapy options can only bring scant survival advantages; thus, the prognosis for EC is very poor. Many EC patients have swallowing difficulties and suffer from significant weight loss; in addition, fasting periods after surgeries are required for most EC patients, resulting in severe nutrition and metabolism problems [2,3]. Malnutrition has been shown to be highly correlated with the occurrence of complications, mortality, and recurrence, as well as organ and immune system dysfunction, in EC patients [4,5]; thus, nutritional support based on the provision of appropriate nutritional risk evaluation is quite important to improve EC patients' prognosis.
Currently, various nutritional screening and assessment tools have been developed and recommended by different organizations, including the Prognostic Nutritional Index (PNI), Abdomen Trauma Index (ATI), Patient-generated Subjective Globe Assessment (PG-SGA), Mini Nutritional Assessment (MNA), Short Form Mini Nutritional Assessment (MNA-SF), Nutrition Risk Screening 2002 (NRS 2002), and Nutrition Risk Index (NRI); however, due to the diversity of patients and diseases, there is no universal gold standard for evaluating nutritional risk. Since nutritional evaluation is commonly demanded for determining an EC patient's treatment, we selected PG-SGA, NRS 2002, and NRI, the most widely accepted nutritional screening tools worldwide, to perform nutritional risk evaluation for a cohort of EC patients in our department. In addition, although studies have shown that albumin is neither specific nor sensitive as a malnutrition marker in many patient populations, it is still recognized that albumin can be considered a reliable parameter for preo-

\footnotetext{
This study was supported by the Science and Technology Planing Project of Guangdong Province (2014A020212541).

${ }^{\S}$ Corresponding Author: Mei Li, Tel. 86-20-61641828, Email. Im198981@126.com

Received: April 23, 2019, Revised: May 28, 2019, Accepted: July 10, 2019

* These authors contributed equally to this study.

This is an Open Access article distributed under the terms of the Creative Commons Attribution Non-Commercial License (http://creativecommons.org/licenses/by-nc/3.0/) which permits unrestricted non-commercial use, distribution, and reproduction in any medium, provided the original work is properly cited.
} 
perative nutrition assessment [6]. Therefore, using albumin as a reference, we compared the clinical performances of PG-SGA, NRS 2002, and NRI in order to determine the most suitable nutritional risk screening tool for EC patients in China.

\section{SUBJECTS AND METHODS}

\section{Patients}

This observational study was conducted with hospitalized patients diagnosed as EC in the Department of Thoracic Surgery, Nanfang Hospital, Southern Medical University, China from July 2016 to December 2017. Initially, a total of 160 EC patients were recruited. The applied exclusion criteria were: $>80$ years old; inability to provide essential information to complete the nutritional evaluation with/without external help; receiving palliative surgery and chemotherapy during the admission period; quitting the survey. As a result, $138 \mathrm{EC}$ patients were enrolled and included in the final analysis. The demographic information for these patients, including age, gender, body mass index (BMI), pathological stage, and tumor differentiation status is summarized and presented in Table 1.

\section{Ethical considerations}

This study was approved by the Southern Medical University Nan Fang Hospital Ethics Committee in accordance with the Helsinki Declaration of 1975, as revised in 2000 (protocol number NFEC-201411-k4-01). The study was also registered in the Chinese Clinical Trial Register with the number CHiCTR19000 22589. Fully written inform consent was obtained from all participants prior to initiating data collection.

\section{Nutrition risk screening}

Table 1. Demographic characteristics for all esophageal cancer (EC) patients

\begin{tabular}{|c|c|c|}
\hline Characteristics & Median (Range) & n (\%) \\
\hline Age (yrs) & $60.59 \pm 7.775(39-78)$ & \\
\hline$<45$ & & $4(2.9)$ \\
\hline $45-65$ & & $100(72.5)$ \\
\hline$>65$ & & $34(24.6)$ \\
\hline \multicolumn{3}{|l|}{ Gender } \\
\hline Male & & $100(72.5)$ \\
\hline Female & & $38(27.5)$ \\
\hline BMl $\left(\mathrm{kg} / \mathrm{m}^{2}\right)$ & $21.51 \pm 2.9798(14.37-29.6)$ & \\
\hline$<18.5$ & & $23(16.7)$ \\
\hline $18.5-23.9$ & & $89(64.5)$ \\
\hline $24.0-27.9$ & & $22(15.9)$ \\
\hline$\geq 28.0$ & & $4(2.9)$ \\
\hline \multicolumn{3}{|l|}{ Pathological stage } \\
\hline 1 & & $18(13.0)$ \\
\hline ॥ & & $58(42.0)$ \\
\hline III & & $62(44.9)$ \\
\hline \multicolumn{3}{|l|}{ Tumor differentiation status } \\
\hline Well differentiated & & $34(24.6)$ \\
\hline Moderately differentiated & & $57(41.3)$ \\
\hline Poorly differentiated & & $45(32.6)$ \\
\hline Unknown & & $2(1.4)$ \\
\hline
\end{tabular}

BMl: body mass index
To evaluate the nutritional condition of the EC patients involved in this study, all participants were analyzed using the NRS 2002, PG-SGA, and NRI tools within 48 hours after hospital admission. The screening was carried out by two independent experienced nurses from the department of thoracic surgery andone supervisor from our Nutrition Unit. On the second day of admission, blood samples were collected, and the serum albumin levels were determined, which were then used as reference levels in the evaluation of the sensitivity and specificity of all screening tools.

The PG-SGA questionnaire was divided into two sections [7]. The first section included body weight history, food intake, nutrition-associated symptoms, activity, and body functions and was completed by the patient or the responsible caregiver. The second section was completed by an experienced nurse and evaluated the patients' fat storage, muscle condition, and edema severity. Based on the results of this comprehensive assessment, a patient's nutritional status was classified as no risk, low risk, or high risk.

The NRS2002 [8] survey contained three parameters, disease severity, nutritional status, and age. The NRS 2002 protocol used in the current study followed that developed by the European Society of Parenteral and Enteral Nutrition (ESPEN) in 2002, except that the body mass index (BMI) threshold was adjusted to 18.5 due to the population difference between European and Chinese patients. In accordance with the standard protocol, when the patient was over 70 years old, one point was added to the total score. Patients with a total score of $\geq 3$ were considered to be at nutritional risk.

The NRI was calculated by using the following formula: 1.519 $\times$ serum albumin level $(\mathrm{g} / \mathrm{L})+0.417 \times$ (current weight/average weight) $\times 100$. An NRI score of $>97.5$ indicates that patient has no risk, scores of 83.5-97.5 indicate low risk, and a score $<83.5$ indicates high nutritional risk [9].

\section{Statistical analysis}

The collected data were revised, coded, tabulated, and introduced into a personal computer. Qualitative data were presented as numbers and percentages. Quantitative data were presented as mean and standard deviation values. All data analysis was performed using SPSS Statistics 17.0 and MedCalc Statistical software. Sensitivity, specificity, and Youden index were calculated to determine the best screening tool for EC patients. The performance evaluation for each tool was determined by using the receiver operating characteristic (ROC) curve. New cut-off points were defined with the Youden index set to the maximum level and were generated automatically by the MedCalc software.

\section{RESULTS}

Demographic information for the participants

Initially, 160 EC patients were selected; however, 5 patients denied participation, 6 patients received chemotherapy, and 11 underwent palliative surgeries. These 22 patients were excluded from the final analysis, resulting in a patient population of 138 and a response rate of $86.25 \%$. The median age for all patients was $60.59 \pm 7.775$ (39-78) years, with a predominance of male 
Table 2. Nutritional risk screening results using different screening tools for EC patients

\begin{tabular}{|c|c|}
\hline Screening tools & n (\%) \\
\hline \multicolumn{2}{|l|}{ PG-SGA } \\
\hline no risk & $4(2.9)$ \\
\hline mildly & $27(19.6)$ \\
\hline severely & 107 (77.5) \\
\hline \multicolumn{2}{|l|}{ NRS 2002} \\
\hline no risk & $60(43.5)$ \\
\hline mildly & $49(35.5)$ \\
\hline severely & $29(21.0)$ \\
\hline \multicolumn{2}{|l|}{$\mathrm{NRI}$} \\
\hline no risk & $80(58.0)$ \\
\hline mildly & $54(39.1)$ \\
\hline severely & $4(2.9)$ \\
\hline
\end{tabular}

PG-SGA, patient-generated subjective globe assessment; NRS 2002, nutrition risk screening 2002; NRI, nutrition risk index.

patients $(72.5 \%, \mathrm{n}=100)$. Most patients' $(64.5 \%)$ body weight was normal, but 26 patients' (18.8\%) BMl was over $24 \mathrm{~kg} / \mathrm{m}^{2}$, which was considered overweight according to the Chinese standard; the remaining patients $(16.7 \%)$ might have nutritional problems as their BMl was less than $18.5 \mathrm{~kg} / \mathrm{m}^{2}$. The proportions at EC pathological stages I, II, and III were $13 \%, 42 \%$, and $44.9 \%$, respectively. Details on the patients' demographic information are presented in Table 1.

Nutrition risk assessment for EC patients

Initially, each of the EC patients' nutrition status was evaluated using PG-SGA, NRS2002, and NRI. The PG-SGA analysis revealed that over $77.5 \%$ of the patients were under severe

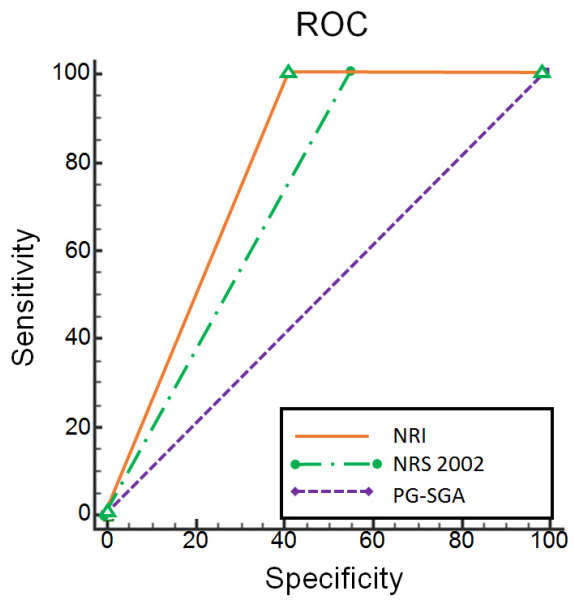

Fig. 1. Comparison of AUROC curves generated from PG-SGA, NRS 2002, and NRI. The AUROC values for PG-SGA, NRS 2002, and NRI were 0.515, 0.722 and 0.796 respectively. AUROC, area under receiver operating characteristic; PG-SGA, patientgenerated subjective globe assessment; NRS 2002, nutrition risk screening 2002; NRI, nutrition risk index.

nutritional risk, but the NRS2002 and NRI screening results suggested only $21.0 \%$ and $2.9 \%$ of the patients, respectively, had severe nutritional problems (Table 2), indicating that even for the same group of patients, using different nutritional assessment tools can produce dramatically different conclusions.

Performance evaluation of different nutritional risk screening tools for EC patients

To compare the results generated by the three different nutritional risk screening tools, we calculated their sensitivity

Table 3. Comparisons of different nutritional risk screening tools

\begin{tabular}{|c|c|c|c|c|c|c|}
\hline & Sensitivity (\%) & Specificity (\%) & $\begin{array}{l}\text { Positive likelihood } \\
\text { ratio }\end{array}$ & $\begin{array}{c}\text { Negative likelihood } \\
\text { ratio }\end{array}$ & Youden index (\%) & AUROC $^{1)}$ \\
\hline \multirow[t]{2}{*}{ PG-SGA } & 100 & 2.96 & 1.03 & 0 & 2.96 & 0.515 \\
\hline & $95 \% C l:[29.2,100]$ & $95 \% C l:[0.8,7.4]$ & & & & \\
\hline \multirow[t]{2}{*}{ NRS 2002} & 100 & 44.44 & 1.8 & 0 & 44.44 & 0.722 \\
\hline & $95 \% C l:[29.2,100]$ & $95 \% \mathrm{Cl}:[35.9,53.2]$ & & & & \\
\hline \multirow[t]{2}{*}{ NRI } & 100 & 59.26 & 2.45 & 0 & 59.26 & 0.796 \\
\hline & $95 \% C l:[29.2,100]$ & $95 \% \mathrm{Cl}:[50.5,67.6]$ & & & & \\
\hline
\end{tabular}

1) AUROC: area under receiver operating characteristic. Method evaluation and comparison were performed using ROC curve analysis.

PG-SGA, patient-generated subjective globe assessment; NRS 2002, nutrition risk screening 2002; NRI, nutrition risk index; Cl, confidence interval.

Table 4. Optimization of nutritional risk screening tools for EC patients.

\begin{tabular}{|c|c|c|c|}
\hline & PG-SGA & NRS 2002 & NRI \\
\hline \multirow[t]{2}{*}{ New cut-off points } & $>15 \quad 1(29)^{*}$ & $>31(50)$ & $\leq 81.6 \quad 1(4)$ \\
\hline & $\leq 15 \quad 0(109)^{\#}$ & $\leq 3 \quad 0(88)$ & $>81.6 \quad 0(134)$ \\
\hline \multirow[t]{2}{*}{ Sensitivity } & $100 \%$ & $100 \%$ & $100 \%$ \\
\hline & $95 \% C l:[29.2,100]$ & $95 \% C l:[29.2,100]$ & 95\%Cl:[29.2, 100] \\
\hline \multirow[t]{2}{*}{ Specificity } & $69.63 \%$ & $65.19 \%$ & $99.26 \%$ \\
\hline & $95 \% \mathrm{Cl}:[61.1,77.2]$ & $95 \% \mathrm{Cl}:[56.5,73.2]$ & 95\%Cl:[95.9, 100] \\
\hline Youden index & $69.63 \%$ & $65.19 \%$ & $99.26 \%$ \\
\hline Positive likelihood ratio & 3.29 & 2.87 & 135 \\
\hline Negative likelihood ratio & 0 & 0 & 0 \\
\hline
\end{tabular}

\footnotetext{
* With nutritional risk

\# Without nutritional risk.

PG-SGA, patient-generated subjective globe assessment; NRS 2002, nutrition risk screening 2002; NRI, nutrition risk index; Cl, confidence interval.
} 
specificity, positive and negative likelihood ratios, Youden index and area under the ROC curve (AUROC) for accuracy evaluation. All three tools achieved $100 \%$ sensitivity, but the NRI appeared to be not only the most specific tool for EC patients with a $59.26 \%$ specificity and a 2.45 positive likelihood ratio, but also it had the greatest accuracy with the highest AUROC value for EC patients (Table 3, Fig. 1).

\section{Optimization of nutritional screening tools for EC patients}

As the initial nutritional risk screening results were not considered satisfactory, we attempted to optimize the three screening tools by re-defining the cut-off points. Therefore, with the AUROC curves generated previously for each screening tool (Fig. 1), we set the respective Youden index to the maximum value with MedCalc and categorized all patients as either with or without nutritional risk. The new cut-off points calculated by MedCalc were 15, 3, and 81.6 for PG-SGA, NRS 2002, and NRI, respectively (Table 4). The sensitivity remained at $100 \%$ for all three tools, but the specificity levels were markedly increased for all three tools. Importantly, our results revealed that NRI achieved the highest specificity, Youden index, and positive likelihood ratio values compared to those for PG-SGA and NRS 2002, suggesting that after optimization, NRI was still the most appropriate nutritional risk screening tool for EC patients.

\section{DISCUSSION}

Many EC patients suffer from other diseases such as diabetes, hypertension, and lung dysfunction. Therefore they may be exposed to multiple drugs which can significantly impair digestion system function. Furthermore, the high consumption burden of the tumor itself, as well as blockage of the esophagus, can severely affect nutrient absorption; therefore, EC patients are usually with high nutritional risk, which can contribute to the poor prognosis for EC patients. As Robinson et al. [10] reported, the incidence of surgery-associated complications was 20 times higher in malnourished EC patients compared to that in patients without nutritional risk.

Various nutritional risk screening tools are widely used in clinical settings to evaluate the nutritional status of patients and provide a reference to decide whether extra nutritional support is required. However, it is recognized that no single nutritional screening tool can be used for all diseases, thus, selecting the most sensitive and accurate nutritional screening tool for patients with a specific disease is highly necessary.

In this study, we chose three classical nutritional screening systems and compared their performance for evaluating the nutritional risk of EC patients. The PG-SGA was developed at the Fox Chase Cancer Center but originated from the Subjective Global Assessment (SGA) tool proposed by Baker et al. [11] in 1982. This system included both self-evaluation and clinician assessment, as well as consideration of metabolic stress, and was reported to be more suitable for cancer patients' nutritional risk evaluation than for other patients [12]; however, a lot of subjective judgment can be involved when using the PG-SGA which can affect the outcome of an evaluation. The NRS 2002 was officially released in 2002 by the European Society of Parenteral and Enteral Nutrition (ESPEN) [13-15] and was first introduced into China in 2005 [16]. The NRS 2002 system integrated patients' nutritional status, severity of the disease, and patient age and was recommended by Chinese Society for Parenteral and Enteral Nutrition (CSPEN), American Society for Parenteral and Enteral Nutrition (ASPEN), and the Society of Critical Care Medicine (SCCM) as the first option for nutritional risk screening $[15,17,18]$. However, it was inapplicable when the patients had significant body weight or food intake variation; thus, it might not be suitable for EC patients. The NRI was first described by Buzby et al. $[19,20]$ in 1988 as a tool to evaluate the severity of surgery-associated complications by measuring serum albumin level and calculating body weight loss percentage, both of which were highly correlated with patients' death rate. The NRI was shown to be more efficient than using albumin or $\mathrm{BMl}$ alone and is more convenient than many other nutritional risk screening tools; hence, its use was recommended by French Programme National Nutrition Sante (FPNNS) to assess nutritional risk for hospitalized patients [21]. However, albumin's half-life is approximately 20 days and is easily affected by stress or pain; in addition, the NRI does not reflect variations in patient age, disease, or food intake and whether the index is appropriate for EC patients has not been previously investigated. Regardless, our results clearly indicate that the NRI was the most suitable nutritional screening tool for EC patients in terms of specificity and accuracy when compared to PG-SGA and NRS 2002.

Paradoxically, we noticed that many EC patients with no nutritional problems received extra nutritional support due to an incorrect nutritional assessment and that additional support did not provide any prognostic advantage. On the other hand, unnecessary nutritional supplementation significantly increased the patients' psychological and economic burden, which should be easily avoided if the nutritional screening tool was properly selected or could be optimized. We, therefore, reset the cut-off points for all three screening tools based on the results of our MedCalc-based analysis. The reset cut-off points did not impair sensitivity, but did markedly increase the specificity and accuracy for all three tools. In addition, the numbers of patients with nutritional risk were significantly reduced, indicating that the use of previously established cut-offs for PG-SGA, NRS 2002 and NRI might identify some EC patients who did not have nutritional problems as being malnourished. Therefore, when evaluating the nutritional status of EC patients, it is necessary to adjust the screening tools' cut-off points. Nevertheless, after optimization of the cut-off points, NRI was still the best nutritional screening tool for EC patients.

To our surprise, our data revealed that fewer Chinese EC patients were at nutritional risk than was expected. We speculate that many EC patients were diagnosed at an early EC stage because of the availability of newly developed EC screening methods [22]; a stage in which their digestion system and food intake were not obviously affected [23]. Moreover, we observed that nearly $20 \%$ of the EC patients enrolled in this study were overweight, according to their BMI values, thus, they did not require additional nutritional supplements. As our study was carried out with a cohort of Chinese EC patients in a singlecenter fashion, whether the NRI would be the most appropriate nutritional screening tool for EC patients in other populations requires further investigation. 


\section{ACKNOWLEDGMENT}

We thank all the colleagues in our department for their kind help and suggestions.

\section{CONFLICT OF INTEREST}

The authors declare that they have no competing interests related to this study.

\section{ORCID}

Wen Dong: https://orcid.org/0000-0003-3955-4062

Xiguang Liu: https://orcid.org/0000-0003-0874-8884

Shunfang Zhu: https://orcid.org/0000-0002-8664-4899

Di Lu: https://orcid.org/0000-0002-8635-6512

KaicanCai: https://orcid.org/0000-0003-4664-6694

RuijunCai: https://orcid.org/0000-0002-2834-2665

Qing Li: https://orcid.org/0000-0002-5938-9167

Jingjing Zeng: https://orcid.org/0000-0001-5386-656X

Mei Li: https://orcid.org/0000-0002-4264-8505

\section{REFERENCES}

1. Xu W, Liu Z, Bao Q, Qian Z. Viruses, other pathogenic microorganisms and esophageal cancer. Gastrointest Tumors 2015;2:2-13.

2. Gupta $\mathrm{R}$, Ihmaidat $\mathrm{H}$. Nutritional effects of oesophageal, gastric and pancreatic carcinoma. Eur J Surg Oncol 2003;29:634-43.

3. Miller KR, Bozeman MC. Nutrition therapy issues in esophageal cancer. Curr Gastroenterol Rep 2012;14:356-66.

4. Sun Z, Cao Z, Ye Q, Liang E, Fu H, Qian X. Preoperative NRS 2002 assessment of nutritional risk of patients with esophagus cancer and postoperative results. Chin J Clin Thorac Cardiovasc Surg 2015;22:323-6.

5. Li Y, Cheng $Y, X u B$, Duan $X, X u$ J, Qi D. The effective of nutrition risk assessment and interventions in patients with esophageal cancer. Chung Hua Hu Li Tsa Chih 2015;50:166-70.

6. Wischmeyer PE, Carli F, Evans DC, Guilbert S, Kozar R, Pryor A, Thiele RH, Everett S, Grocott M, Gan TJ, Shaw AD, Thacker JK, Miller TE, Hedrick TL, McEvoy MD, Mythen MG, Bergamaschi R, Gupta R, Holubar SD, Senagore AJ, Abola RE, Bennett-Guerrero E, Kent ML, Feldman LS, Fiore JF Jr; Perioperative Quality Initiative (POQI) 2 Workgroup. American society for enhanced recovery and perioperative quality initiative joint consensus statement on nutrition screening and therapy within a surgical enhanced recovery pathway. Anesth Analg 2018;126:1883-95.

7. $\mathrm{Hu} C L, Y u$ M, Yuan $K T$, Yu HL, Shi YY, Yang JJ, Li W, Jiang HP, Li ZN, Xu HX, Ba Y, Wang KH, Li SY, Shi HP. Determinants and nutritional assessment value of hand grip strength in patients hospitalized with cancer. Asia Pac J Clin Nutr 2018;27:777-84.

8. Kondrup J, Rasmussen $\mathrm{HH}$, Hamberg O, Stanga Z; Ad Hoc ESPEN Working Group. Nutritional risk screening (NRS 2002): a new method based on an analysis of controlled clinical trials. Clin Nutr 2003;22:321-36.
9. Oh CA, Kim DH, Oh SJ, Choi MG, Noh JH, Sohn TS, Bae JM, Kim S. Nutritional risk index as a predictor of postoperative wound complications after gastrectomy. World J Gastroenterol 2012;18: 673-8.

10. Robinson MK, Trujillo EB, Mogensen KM, Rounds J, McManus K, Jacobs DO. Improving nutritional screening of hospitalized patients: the role of prealbumin. JPEN J Parenter Enteral Nutr 2003;27:389-95.

11. Makhija S, Baker J. The Subjective Global Assessment: a review of its use in clinical practice. Nutr Clin Pract 2008;23:405-9.

12. Bauer J, Capra S, Ferguson M. Use of the scored Patient-Generated Subjective Global Assessment (PG-SGA) as a nutrition assessment tool in patients with cancer. Eur J Clin Nutr 2002;56:779-85.

13. Rasmussen $\mathrm{HH}$, Kondrup J, Ladefoged K, Staun M. Clinical nutrition in danish hospitals: a questionnaire-based investigation among doctors and nurses. Clin Nutr 1999;18:153-8.

14. Kondrup J, Rasmussen $\mathrm{HH}$, Hamberg $\mathrm{O}$, Stanga $\mathrm{Z}$; Ad Hoc ESPEN Working Group. Nutritional risk screening (NRS 2002): a new method based on an analysis of controlled clinical trials. Clin Nutr 2003;22:321-36

15. Chinese Medical Association. Nutrition support guidelines for clinical diagnosis and treatment. Beijing: People's medical Publishing House Co.; 2009. p. 8-9.

16. Chen W, Jiang Z, Zhang $Y$, Wang $X$, Chen $C$, Shi $Y$. Evaluation of European nutrition risk screening method in Chinese hospitalized patients practice. Chin J Clin Nutr 2005;13:137-41.

17. Kondrup J, Allison SP, Elia M, Vellas B, Plauth M; Educational and Clinical Practice Committee, European Society of Parenteral and Enteral Nutrition (ESPEN). ESPEN guidelines for nutrition screening 2002. Clin Nutr 2003;22:415-21.

18. McClave SA, Taylor BE, Martindale RG, Warren MM, Johnson DR Braunschweig C, McCarthy MS, Davanos E, Rice TW, Cresci GA, Gervasio JM, Sacks GS, Roberts PR, Compher C; Society of Critical Care Medicine; American Society for Parenteral and Enteral Nutrition. Guidelines for the provision and assessment of nutrition support therapy in the adult critically ill patient: society of critical care medicine (SCCM) and American society for parenteral and enteral nutrition (A.S.P.E.N.). JPEN J Parenter Enteral Nutr 2016;40: 159-211.

19. Buzby GP, Knox LS, Crosby LO, Eisenberg JM, Haakenson CM, McNeal GE, Page CP, Peterson OL, Reinhardt GF, Williford WO. Study protocol: a randomized clinical trial of total parenteral nutrition in malnourished surgical patients. Am J Clin Nutr 1988;47:366-81.

20. Buzby GP, Williford WO, Peterson OL, Crosby LO, Page CP, Reinhardt GF, Mullen JL. A randomized clinical trial of total parenteral nutrition in malnourished surgical patients: the rationale and impact of previous clinical trials and pilot study on protocol design. Am Clin Nutr 1988;47:357-65.

21. Hasselmann M, Alix E. Tools and procedures for screening for malnutrition and its associated in risks in hospital. Nutr Clin Metab 2003; 17:218-26

22. Meves V, Behrens A, Pohl J. Diagnostics and early diagnosis of esophageal cancer. Viszeralmedizin 2015;31:315-8.

23. Reim $\mathrm{D}$, Friess $\mathrm{H}$. Feeding challenges in patients with esophageal and gastroesophageal cancers. Gastrointest Tumors 2016;2:166-77. 